\title{
Práctica clínica de otorrinolaringólogos en Chile durante la pandemia COVID-19
}

\section{Clinical practices of otolaryngologists in Chile during the COVID-19 pandemic}

\author{
Galia Villarroel O. ${ }^{1}$, Constanza Valdés P., ${ }^{2,3}$, Natalia Tamblay N. ${ }^{3}$, Felipe Cardemil M. ${ }^{2,3}$
}

'Facultad de Medicina, Universidad de Chile. Santiago, Chile.

${ }^{2}$ Servicio de

Otorrinolaringología, Hospital del Salvador. Santiago, Chile.

${ }^{3}$ Departamento de Otorrinolaringología, Facultad de Medicina, Universidad de Chile. Santiago, Chile.

Los autores declaran no tener conflictos de interés.

Recibido el 16 de marzo de 2021. Aceptado el 28 de julio de 2021

Correspondencia: Felipe Cardemil M Estoril 450, Las Condes. Santiago, Chile

Email: felipecardemil@med. uchile.cl

\section{Resumen}

Introducción: La práctica otorrinolaringológica presenta un riesgo elevado de contagio de SARS-CoV-2. Considerando esto, diversas sociedades científicas a nivel mundial llamaron a establecer prioridades en la atención clínica. Objetivo: Evaluar el impacto de la pandemia en la práctica clínica y quirúrgica de los otorrinolaringólogos en Chile, así como su impacto económico durante el inicio de la pandemia. Material y Método: Estudio de corte transversal, utilizando un cuestionario autoadministrado en línea a los socios de la Sociedad Chilena de Otorrinolaringología. Resultados: De un total de 461 socios, se obtuvieron 214 respuestas (46,4\%). Un 66\% en el servicio público y un $57 \%$ en el sistema privado refirió una disminución de la actividad de consulta ambulatoria en $>75 \%$, o atención sólo urgencias. Un $92 \%$ en el servicio público y un $96 \%$ en el sistema privado declaró una reducción de la actividad quirúrgica en $>75 \%$, o cirugías sólo oncológica o urgencias. La prevalencia de autorreporte de PCR SARS-CoV-2 positiva fue de un $5,1 \%$ de los encuestados. En cuanto al impacto económico, un $63 \%$ tuvo una disminución de al menos la mitad de sus ingresos y un $36,9 \%$ tuvo que recurrir a alguna ayuda financiera. Conclusión: Durante el inicio de la pandemia hubo un gran impacto a nivel nacional en la práctica clínica otorrinolaringológica, esto se observó en la consulta ambulatoria, en los procedimientos quirúrgicos y también en el ámbito económico. Estos hallazgos fueron comparables con estudios en otros países.

Palabras clave: Otorrinolaringología, COVID-19, práctica clínica, pandemia.

\section{Abstract}

Introduction: Otolaryngologists have significantly higher risk of COVID-19 infection due to the nature of the specialty. Given the above, various scientific societies worldwide called to prioritize clinical care. Aim: Evaluate the impact of the pandemic on the clinical and surgical practice of otolaryngologists in Chile, alongside with the economic impact at the beginning of the pandemic. Material and Method: Cross-sectional study with an online self-administered questionnaire to members of the Chilean Society of Otorhinolaryngology. Results: From a total of 461 members, 214 responses were obtained (46.4\%); Sixty six percent in the public service and fifty seven percent in the private system reported a decrease in ambulatory consultation activity by $>75 \%$ or only emergency care. Ninety two percent in the public service and ninety six percent in the private system reported a reduction in surgical activity by $>75 \%$ or only oncology/emergency care. The prevalence of self-report infection with positive PCR for SARS-CoV-2 was 5.1\%. Regarding the economic impact, $63 \%$ had a decrease of at least half of their income and $36.9 \%$ had to resort to some financial aid. Conclusion: During the pandemic, there was a great impact at the national level in the otolaryngological clinical practice, both in the outpatient consultation and in surgical procedures, as well as in the economic sphere. These findings were similar with studies in other countries.

Keywords: Otolaryngology, COVID-19, clinical practice, pandemic. 


\section{Introducción}

En diciembre de 2019, un brote de neumonía producido por un nuevo coronavirus se reportó en Wuhan, China, el SARS-CoV-2 ${ }^{1}$. La enfermedad causada por este virus se denominó por consenso internacional COVID-19, y se ha extendido rápidamente por el mundo desde entonces. La OMS lo reconoció como una pandemia global el 11 de marzo de 2020, afectando a más de 80.000 .000 de personas y provocando más de 1.800.000 muertes en el mundo al término de $2020^{2,3}$.

Los síntomas de COVID-19 incluyen fiebre, tos, cefalea, odinofagia, congestión nasal, fatiga y mialgias ${ }^{4,5}$, también se ha reportado anosmia y disgeusia como síntomas prevalentes ${ }^{6,7}$. Pacientes con estos síntomas se presentan de forma frecuente en los servicios de otorrinolaringología (ORL). Si bien la enfermedad se transmite principalmente a través de gotitas, se ha demostrado que el SARS-CoV-2 permanece viable en aerosoles durante al menos tres horas ${ }^{8}$.

Los trabajadores de la salud representan entre el $3,8 \%$ y $24,1 \%$ de la población infectada en China ${ }^{9,10}$, Estados Unidos de Norteamérica (EE. UU. ${ }^{11}$, Italia ${ }^{12}$ y España ${ }^{13}$; alrededor del $15 \%$ de ellos desarrolló enfermedad grave con una letalidad de $0,1 \%$ a $1,1 \%$. Se plantea que los otorrinolaringólogos se encuentran particularmente en riesgo de contagio dado la alta carga viral en el tracto aerodigestivo superior y el riesgo de aerosolización inherente a los procedimientos otorrinolaringológicos comunes y la manipulación quirúrgica de la vía aérea ${ }^{14,15}$.

A medida que los sistemas de salud se vieron enfrentados a un número creciente de casos, con el objetivo de proteger tanto a los pacientes como al personal de salud, además de preservar los elementos de protección personal (EPP), se fue generando un cambio en la gestión de atención de salud, orientándose principalmente a la atención de pacientes infectados por SARS-CoV-2 y optimizar recursos en un momento de crisis.

Dado lo anterior, múltiples sociedades científicas y departamentos de ORL a lo largo del mundo recomendaron limitar la atención clínica en este momento a problemas urgentes y tiempo dependientes, limitar los procedimientos productores de aerosoles y posponer las cirugías electivas hasta el paso de la pandemia ${ }^{16-24}$.

En Chile, se decretó alerta sanitaria y se le otorgó a la Subsecretaría de Redes Asistenciales la facultad para coordinar la Red Asistencial del país, tanto de prestadores públicos como privados. El 27 de marzo de 2020 , esta entidad indicó postergar la atención ambulatoria, siempre y cuando esto no constituyera un riesgo para el paciente, redistribuir los horarios de los médicos para dar prioridad a la atención de pacientes hospitalizados y de urgencia y suspender las cirugías electivas ${ }^{25,26}$.

\section{Objetivo}

Evaluar el impacto de la pandemia en la práctica clínica y quirúrgica de los otorrinolaringólogos en Chile, así como también su impacto económico.

\section{Material y Método}

Se realizó un estudio de corte transversal mediante un cuestionario autoadministrado en línea a través de la plataforma Google Forms a los otorrinolaringólogos y residentes de otorrinolaringología socios de la Sociedad Chilena de Otorrinolaringología, Medicina y Cirugía de Cabeza y Cuello (SOCHIORL).

La encuesta constaba de 17 preguntas en cuatro secciones (Tabla 1): caracterización de la muestra, carga asistencial, modificaciones en la labor asistencial e impacto económico. Fue difundida a través de la lista de correos electrónicos en junio de 2020, preguntando respecto al período de abril y mayo de 2020 . Se envió en tres oportunidades para aumentar la tasa de respuesta y se incorporó un sistema para evitar respuestas dobles, resguardando el anonimato. Se resguardó la confidencialidad de las opiniones obtenidas.

Se realizó un análisis descriptivo y estadístico con STATA 12. Se realizó descripción de variables cualitativas con frecuencias absolutas y relativas, variables cuantitativas con medidas de tendencia central. 


\section{ARTÍCULO DE INVESTIGACIÓN}

\section{Tabla 1. Encuesta de opinión}

\section{Caracterización de la muestra}

1.1 Género

1.2 Edad

1.3 Región y ciudad de residencia

1.4 ¿En qué etapa de la carrera se encuentra? Residente ORL

$\mathrm{ORL}<5$ años experiencia laboral

ORL 6 a 15 años de experiencia laboral

ORL > 15 años de experiencia laboral

1.5 ¿En qué centro asistencial trabaja? Marque todas las alternativas que aplican:

Hospital de la red pública de atención

Hospital Universitario

Hospital de Fuerzas Armadas

Hospital de seguridad laboral

Otro sistema público (APS, UAPORRINO, nivel secundario, etc.)

Consulta en el sistema privado de salud (consulta particular o clínica)

1.6 En caso de trabajar en hospital (alternativa 1-4 de pregunta anterior) ¿Cuántas horas a la semana trabaja en la institución hospitalaria (excluyendo consulta privada) donde se desempeña principalmente?

No trabajo en hospital

Jornada $11 \mathrm{~h} /$ semana

Jornada $22 \mathrm{~h} /$ semana

Jornada $33 \mathrm{~h} /$ semana

Jornada $44 \mathrm{~h} /$ semana

\section{Carga asistencial}

2.1 ¿En qué porcentaje se ha reducido su actividad de consulta ambulatoria presencial de otorrinolaringología?

Sistema público

No trabajo en este tipo de establecimiento

No ha disminuido mi consulta

Disminuyó en menos de un $25 \%$

Disminuyó en un 25\% a 50\%

Disminuyó en un $50 \%$ a $75 \%$

Disminuyó en más de un $75 \%$

Estoy atendiendo sólo urgencias

\section{Sistema privado}

No trabajo en este tipo de establecimiento

No ha disminuido mi consulta

Disminuyó en menos de un $25 \%$

Disminuyó en un $25 \%$ a $50 \%$

Disminuyó en un $50 \%$ a $75 \%$

Disminuyó en más de un $75 \%$

Estoy atendiendo sólo urgencias

2.2 ¿En qué porcentaje se ha reducido su actividad quirúrgica?

Sistema público

No trabajo en este tipo de establecimiento

No ha disminuido mi práctica quirúrgica

Disminuyó en menos de un $25 \%$

Disminuyó en un $25 \%$ a $50 \%$

Disminuyó en un $50 \%$ a $75 \%$

Disminuyó en más de un $75 \%$

Sólo se intervienen pacientes oncológicos y de urgencia

\section{Sistema privado}

No trabajo en este tipo de establecimiento

No ha disminuido mi práctica quirúrgica

Disminuyó en menos de un $25 \%$

Disminuyó en un $25 \%$ a $50 \%$

Disminuyó en un $50 \%$ a $75 \%$

Disminuyó en más de un $75 \%$

Sólo se intervienen pacientes oncológicos y de urgencia

2.3 ¿Cuál es su modalidad de trabajo actual? (puede marcar más de una opción)

Sistema público

No trabajo en este tipo de establecimiento

Presencial

Teletrabajo

Suspendí mis actividades por la pandemia
Sistema privado

No trabajo en este tipo de establecimiento

Presencial

Teletrabajo

Suspendí mis actividades por la pandemia 


\section{Modificaciones en la labor asistencial}

3.1 ¿En la institución en la que trabajo como otorrinolaringólogo me han solicitado que realice turnos de pacientes hospitalizados y/o urgencias de otorrinolaringología en pacientes COVID-19 (+)? Ej.: traqueostomía o epistaxis en paciente COVID-19 (+).

Sí

No

3.2 Durante la pandemia, ¿le han asignado realizar labores clínicas asistenciales diferentes a la especialidad de otorrinolaringología?

Sí, por instrucción de jefatura

Sí, por voluntariado

No

3.3 ¿Ha estado en cuarentena preventiva a razón de exposición laboral a COVID-19? Sí

No

3.4 ¿Se ha contagiado de COVID-19 confirmado con PCR?

Sí

No

\section{Impacto económico}

4.1 ¿En qué porcentaje disminuyó su ingreso promedio mensual en abril y mayo del año 2020 producto de la pandemia con respecto al mismo período del año 2019?

Mis ingresos no han disminuido

Mi ingreso mensual disminuyó en menos de un 25\%

Mi ingreso mensual disminuyó en un 25\% a 50\%

Mi ingreso mensual disminuyó en un 50\% a 75\%

Mi ingreso mensual disminuyó en más de un 75\%

Disminuyó en un $100 \%$

4.2 Respecto a mi carga laboral habitual previo a la pandemia, cuando la situación sanitaria se normalice, mi intención es: Retomarla al mismo nivel prepandemia

Disminuirla para pasar más tiempo con mi familia, hobbies u otras actividades

Aumentarla para recuperar mi situación financiera o habilidad quirúrgica

4.3 ¿Ha tenido que recurrir a alguna ayuda financiera durante la pandemia como solicitud de crédito de consumo, repactación de crédito, postergación del pago de cuotas, entre otros?

Sí

No

4.4 ¿Cuántas personas dependen económicamente de usted?

*ORL: otorrinolaringología. APS: atención primaria de salud. UAPORRINO: Unidad de Atención Primaria Otorrino

\section{Resultados}

\section{Caracterización de la muestra}

De un total de 461 socios se obtuvieron 214 respuestas $(46,4 \%)$, siendo el $62,1 \%$ de sexo masculino. El promedio de edad fue de 45,8 años $\pm 12,6$, con un rango de 25 a 73 años. Con respecto al lugar de residencia, lo más frecuente fue Santiago (65,9\%), seguido por Concepción (7,5\%), Viña del Mar (4,7\%) y Temuco (2,8\%).
La Tabla 2 muestra la distribución de la etapa de la carrera de los encuestados, donde el $44,4 \%$ eran otorrinolaringólogos con más de 15 años de experiencia laboral, seguido de un $23,4 \%$ con 6 a 15 años de experiencia, un $18,7 \%$ con menos de 5 años de experiencia laboral y por último un 13,6\% de residentes.

En cuanto a los centros asistenciales donde se desempeñaban profesionalmente, exclusiva o parcialmente, un $74,8 \%$ declaró trabajar en 


\begin{tabular}{|lcc|}
\hline \multicolumn{4}{l}{ Tabla 2. Distribución etapa de la carrera } \\
\hline Etapa de la carrera & Frecuencia & $\%$ \\
\hline Residentes & 29 & 13,6 \\
Menos de 5 años de experiencia laboral & 40 & 18,7 \\
6 a 15 años de experiencia laboral & 50 & 23,4 \\
Más de 15 años de experiencia laboral & 95 & 44,4 \\
Total & 214 & 100 \\
\hline
\end{tabular}

el sistema privado de salud y un 51,4\% en algún hospital de la red de atención pública. El $21 \%$ trabajaba en un hospital universitario, el $5,1 \%$ en otro tipo de establecimiento. En caso de trabajar en hospital (red pública, hospital universitario, fuerzas armadas o de seguridad laboral), la distribución de jornada laboral en la institución donde se desempeñaban principalmente fue: jornada 11 horas/semana $14,8 \%$; 22 horas/semana: 46,5\%; 33 horas/semana 7\%; 44 horas/semana $31,7 \%$.

\section{Carga asistencial}

Las preguntas se realizaron con respecto a los cambios de la actividad clínica de los meses de abril y mayo de 2020 en comparación con el mismo periodo del año anterior.

2.1. Reducción de la actividad de consulta ambulatoria presencial de ORL (Figura 1): Se observó que un $66 \%$ de los encuestados que trabajaban en el servicio público y un 57\% de los que trabajaban en el servicio privado, refirió una disminución de la consulta ambulatoria en más de un $75 \%$ o que se encontraban atendiendo sólo urgencias.

2.2. Reducción de la actividad quirúrgica (Figura 2): Un 92\% de los encuestados que trabajaban en el sistema público y un $96 \%$ de los que trabajaban en el sistema privado, refirió disminución de la práctica quirúrgica en más de un $75 \%$ o que se encontraba operando sólo pacientes oncológicos/urgencias.

2.3. Modalidad de trabajo (Figura 3): Se preguntó sobre la modalidad de trabajo, es decir, si se encontraban realizando trabajo presencial, teletrabajo, ambas modalidades o si habían suspendido las actividades laborales. Un 84\% de los encuestados se encontraba realizando trabajo presencial en el sistema público en contraste con un $74 \%$ en el sistema privado. Un $41 \%$ se encontraba realizando teletrabajo en el sistema público y un $28 \%$ en el sistema privado. Por otro lado, al preguntar por la suspensión completa de actividades laborales, un $5 \%$ de los que trabajan en el sistema público habían interrumpido sus actividades en comparación con un $20 \%$ en el sistema privado. No se preguntó de forma dirigida las razones de elegir cada modalidad de trabajo.

\section{Modificaciones de la labor asistencial producto de la pandemia}

3.1. Atención ORL de pacientes COVID-19 (+): Un $64,5 \%$ de los encuestados atendió pacientes COVID-19 (+) durante turnos de pacientes hospitalizados y/o urgencias de otorrinolaringología.

3.2. Labores asistenciales diferentes a la especialidad: El 43,9\% de los encuestados realizó labores diferentes a la especialidad de otorrinolaringología. De éstos, el 50\% fue por voluntariado, el $45 \%$ por instrucción de jefatura y el $5 \%$ por ambos motivos.

3.3. Cuarentena preventiva: el $15,4 \%$ tuvo que realizar cuarentena preventiva a razón de exposición laboral a COVID-19.

3.4. La prevalencia de contagio conocido de SARS-CoV-2 confirmado con PCR de los otorrinolaringólogos encuestados fue de un 5,1\%.

\section{Impacto económico}

4.1. Reducción ingreso mensual (Figura 4): La Figura 4 muestra la disminución del ingreso mensual de los meses de abril y mayo de 2020 en comparación con el mismo período del año anterior por autorreporte de los encuestados. Un 63\% del total de los encuestados refirió una disminución del $50 \%$ o más de su ingreso mensual. Al analizar a los encuestados que trabajaban de forma exclusiva o parcial en el sistema privado, es decir, de forma independiente, se observó que un $77 \%$ refirió una disminución del $50 \%$ o más de su ingreso mensual.

4.2. Carga laboral luego de la pandemia (Tabla 3): Una vez que termine la pandemia, un $23,4 \%$ de los encuestados desea disminuir su carga laboral, un 59,8\% mantener su carga laboral habitual y un $16,8 \%$ deseaba aumentarla. Al estratificar este deseo con la disminución de ingresos, observamos que, a mayor disminución de ingresos, mayor proporción 

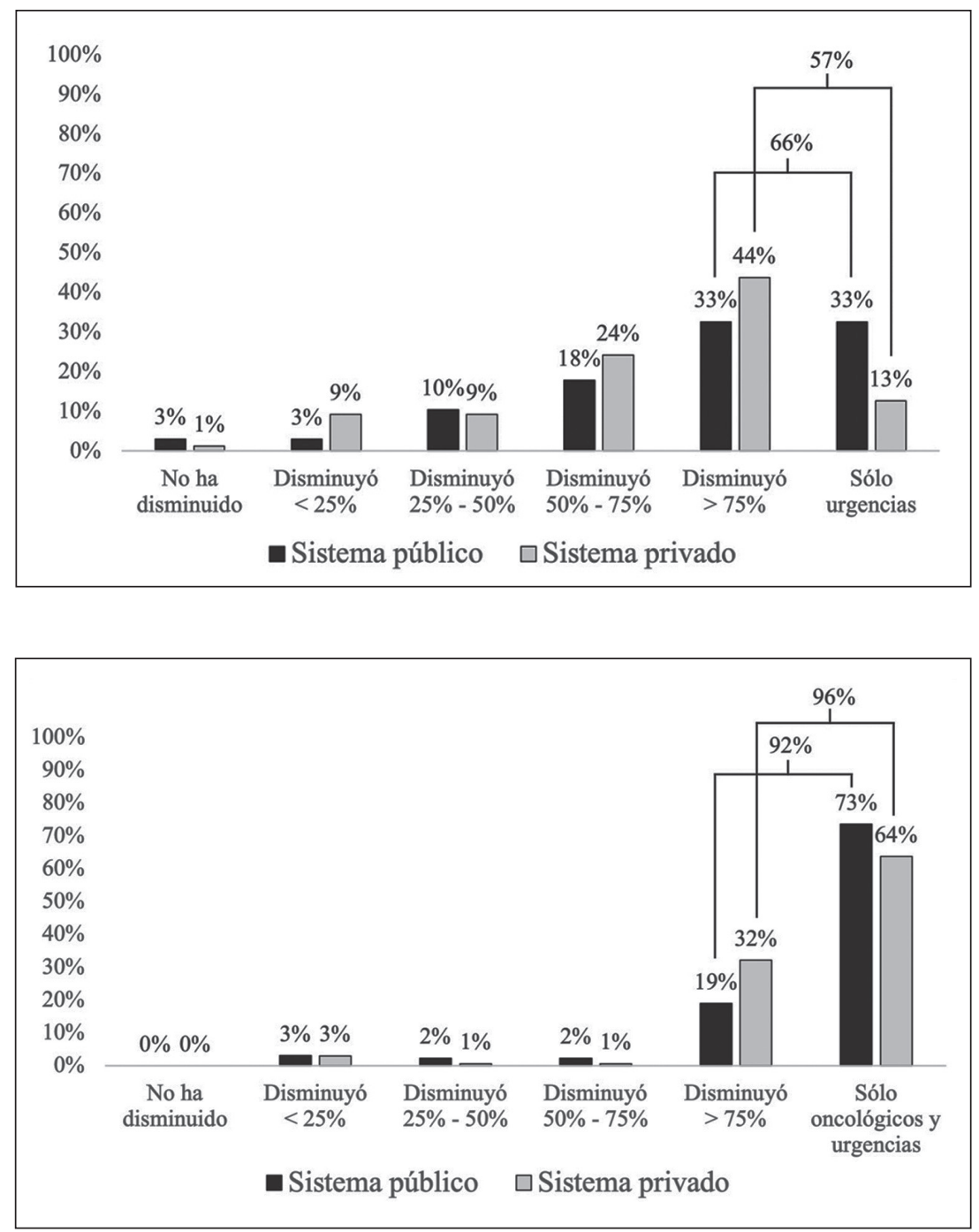

Figura 1. Disminución actividad en consulta ambulatoria presencial otorrinolaringológica meses abril y mayo de 2020, con respecto al mismo período del año 2019.

Figura 2. Disminución actividad quirúrgica otorrinolaringológica meses abril y mayo de 2020, con respecto al mismo período del año 2019.

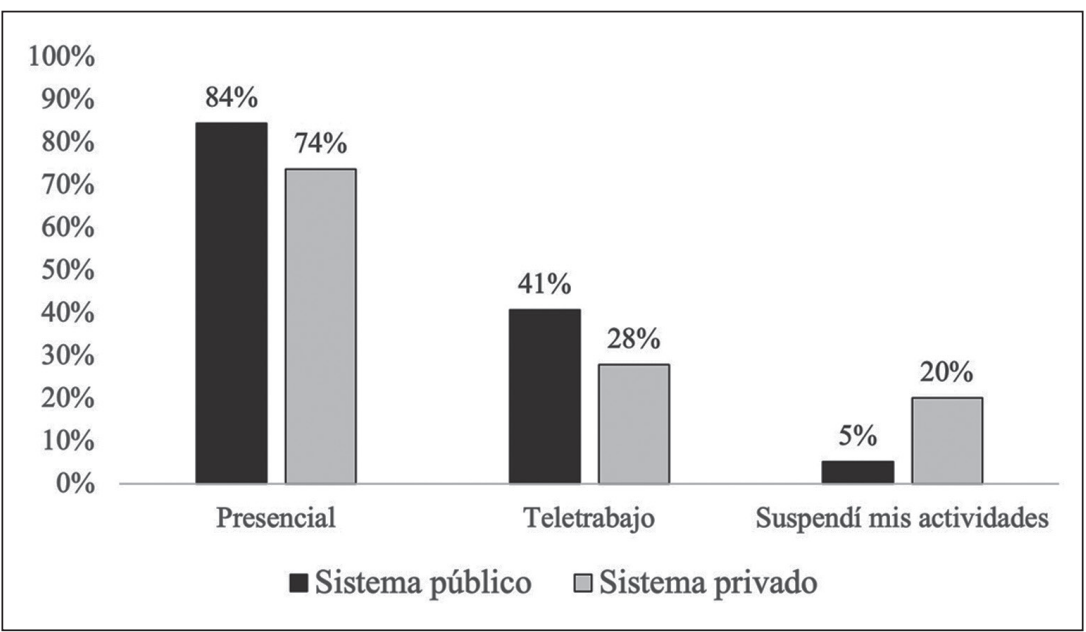

Figura 3. Modalidad de trabajo sistema público y privado. 


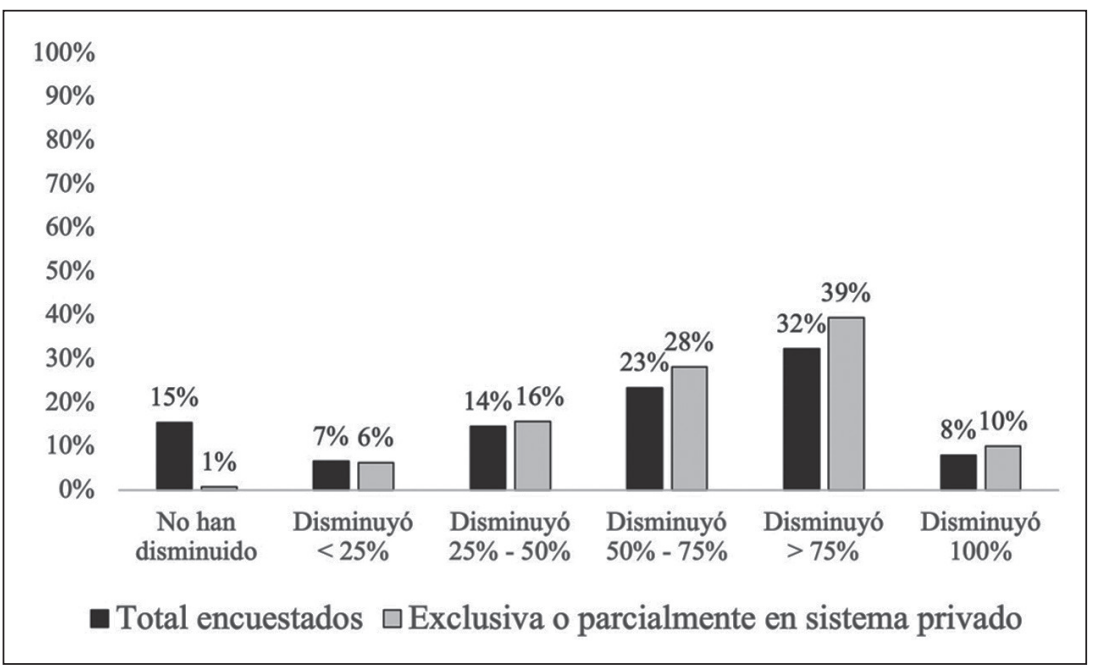

Figura 4. Disminución de ingreso mensual meses abril y mayo de 2020 , con respecto al mismo período del año 2019.

\begin{tabular}{|lccc|}
\hline \multicolumn{3}{|c|}{ Tabla 3. Disminución de ingresos y carga laboral } \\
\hline Ingresos & Disminuir & Mantener & Aumentar \\
\hline 0 & $12,50 \%$ & $6 \%$ & $38,89 \%$ \\
\hline$<25 \%$ & $6,25 \%$ & $8 \%$ & $5,56 \%$ \\
\hline $25 \%-50 \%$ & $15,62 \%$ & $14 \%$ & $11,11 \%$ \\
\hline $50 \%-75 \%$ & $25 \%$ & $24 \%$ & $16,67 \%$ \\
$>75 \%$ & $33,59 \%$ & $34 \%$ & $25 \%$ \\
\hline $100 \%$ & $7,03 \%$ & $14 \%$ & $2,78 \%$ \\
\hline Total & $100 \%$ & $100 \%$ & $100 \%$ \\
\hline
\end{tabular}

de encuestados deseaban aumentar su carga laboral en el futuro.

4.3. Ayuda financiera: un $36,9 \%$ tuvo que recurrir a alguna ayuda financiera durante la pandemia. Se realizó una regresión logística considerando como variable dependiente el pedir ayuda financiera, controlando por género, etapa de la carrera y contagio por SARS-CoV-2 (Tabla 4). Las variables que se asociaron de manera independiente a la necesidad de ayuda financiera fueron la cantidad de personas que dependen económicamente del profesional (odds ratio [OR] 1,37; intervalo de confianza [IC] 95\%: 1,099 - 1,720; $\mathrm{p}=0,005)$,

\begin{tabular}{lccc}
\multicolumn{4}{l}{ Tabla 4. Regresión logística multivariada para variable necesidad de solicitud de apoyo financiero } \\
\hline Variable & OR & valor de $\mathbf{p}$ & IC95\% \\
Dependencia económica & 1,37 & 0,005 & $1,099-1,720$ \\
Ingresos & 1,34 & 0,027 & $1,035-1,751$ \\
\hline Edad & 0,95 & 0,031 & $0,919-0,996$ \\
Género & 1,11 & 0,744 & $0,568-2,206$ \\
\hline Etapa & 1,23 & 0,294 & $0,834-1,816$ \\
Contagio SARS-CoV-2 & 1,79 & 0,397 & $0,461-7,009$ \\
\hline OR: Odds ratio; IC 95\%: intervalo de confianza 95\%.
\end{tabular}


la magnitud de la disminución de los ingresos (OR: 1,34; IC 95\%: $1,035-1,751 ; \mathrm{p}=0,027$ ) y la edad (OR: 0,95; IC 95\%: 0,919 - 0,996; $\mathrm{p}=0,031)$.

\section{Discusión}

La pandemia por COVID-19 ha llevado a un cambio drástico en nuestro diario vivir. Los sistemas de salud son los principales involucrados, con esfuerzos monumentales con el objetivo de resguardar la salud tanto de los pacientes como la de los profesionales de la salud. Una gran preocupación con respecto al riesgo de contagio por SARS-CoV-2 se observó en la comunidad otorrinolaringológica a lo largo del mundo, dado la naturaleza de la práctica clínica de esta especialidad. Si bien diferentes instituciones crearon guías con recomendaciones para la atención de pacientes otorrinolaringológicos en la pandemia, se observó una variabilidad en la puesta en acción de estas a lo largo del mundo ${ }^{22,27}$. En este estudio podemos observar el gran impacto de la pandemia en la práctica clínica otorrinolaringológica en Chile, con una gran disminución de la atención ambulatoria y actividad quirúrgica, tanto en el sistema público como privado, sin embargo, la suspensión total de actividades estuvo presente en un mayor porcentaje en el sistema privado.

Una encuesta realizada por la Sociedad Española de Otorrinolaringología y Cirugía de Cabeza y Cuello (SEORL), mostró una mayor proporción de otorrinolaringólogos $(70,6 \%)$ que refirieron una reducción de la consulta ambulatoria en $>75 \%$ o atención sólo de urgencias, en comparación a lo encontrado en nuestro estudio (66\% sistema público y $57 \%$ sistema privado ${ }^{28}$. En la división de otorrinolaringología de la Facultad de Medicina de Yale, EE. UU., toda la atención no urgente fue cancelada, reagendada o realizada vía telemedicina, observándose una disminución de la consulta ambulatoria en un $82 \%$ en marzo de 2020 con respecto al mismo periodo de $2019^{29}$.

En cuanto al ámbito quirúrgico, en nuestro estudio observamos que un $92 \%$ de los encuestados que trabajaban en el sistema público y un $96 \%$ de los que trabajaban en el sistema privado, refirieron una disminución del $75 \%$ o más de la práctica quirúrgica o que se encon- traban operando sólo pacientes oncológicos/ urgencias, en contraste con el $78 \%$ observado en la encuesta realizada por la SEORL ${ }^{28}$. Un estudio italiano por Manneli y cols. ${ }^{30}$ realizó una encuesta a nivel nacional, observando una reducción del 89,91\% de cirugías ambulatorias, y del $83,28 \%$ de cirugías no ambulatorias con respecto al año anterior. Entre los diferentes tipos de cirugía, los que se vieron menos afectados por la pandemia fueron las cirugías oncológicas y las de emergencia, con un 10,74\% y $3,54 \%$, respectivamente. Por otro lado, la gran mayoría declaró una reducción drástica de los procedimientos endoscópicos rinosinusales $(98,53 \%)$, cirugía de otorrinolaringología pediátrica $(97,59 \%)$, de oído $(94,90 \%)$ y procedimientos quirúrgicos electivos (100\%).

Resulta difícil comparar este impacto dado las diferentes políticas públicas instauradas en cada país. En Chile, en el período en que se realizó la encuesta, se instauró la postergación de todas las cirugías electivas cuyo retraso no signifique un riesgo grave para la salud del paciente, asociado a cuarentenas a lo largo del país ${ }^{31}$, lo cual explicaría la drástica reducción de las cirugías en nuestro estudio. La disminución o suspensión de la actividad quirúrgica trae consigo múltiples consecuencias, principalmente en la patología oncológica, llevando a peores resultados, a requerir tratamientos más agresivos con una morbilidad agregada y aumento de la mortalidad ${ }^{32}$.

De las consultas realizadas en la división de otorrinolaringología de la Facultad de Medicina de Yale ${ }^{29}$, destaca que el 55,8\% se realizó vía telemedicina, el año anterior no se habían realizado consultas en esta modalidad. Si bien en nuestro estudio no tenemos comparación temporal de la proporción de médicos realizando telemedicina previo a la pandemia, actualmente un $41 \%$ de los encuestados se encuentra realizando esta modalidad de trabajo en el sistema público y un $28 \%$ en el sistema privado. La telemedicina es un recurso en expansión, particularmente en el contexto de priorización de la atención durante la pandemia por COVID-19. Una revisión sistemática por Ning y cols. ${ }^{33}$, respalda la prestación de atención otorrinolaringológica vía telemedicina, ya que estos modelos presentan altas tasas de satisfacción del paciente y del proveedor, producen imágenes adecuadas para el diag- 
nóstico y son capaces de producir altas tasas de concordancia diagnóstica con evaluaciones en persona.

La reestructuración del sistema de salud para combatir la pandemia ha sido un desafío permanente, dentro de las estrategias disponibles se destinó al personal a otras unidades. En nuestro estudio un $43,9 \%$ de los encuestados tuvo que realizar labores diferentes a la especialidad de otorrinolaringología, lo cual podría significar un factor de estrés para quienes tuvieron que realizar estas labores. En Italia cerca de un $70 \%$ declaró una reasignación de miembros del equipo de otorrinolaringología a otros departamentos durante la pandemia ${ }^{30}$.

Destaca en nuestro estudio el bajo autorreporte de PCR SARS-CoV-2, con un 5\% de positividad. Al comparar con otras series, en Italia ${ }^{30}$ se observó un $20 \%$ de positividad, llegando hasta $34 \%$ en las zonas más afectadas. Otro estudio italiano evaluó la serología en todo el personal de un departamento de otorrinolaringología, observando un 9\% de positividad y la única variable asociada con un mayor riesgo de infección fue el número de contactos extrahospitalarios sin $\mathrm{EPP}^{34}$. Es difícil interpretar el bajo porcentaje de contagio en nuestra muestra. Una de las hipótesis es que corresponde a un autorreporte, por lo cual podría estar sujeto a sesgo de información; otra explicación podría ser que esta encuesta se realizó a tres meses de declarada la pandemia en Chile, por lo que era aún reciente; por último, el miedo al contagio, al considerarse una especialidad en riesgo, podría haber favorecido un mayor uso de EPP para aerosoles dentro de los otorrinolaringólogos en Chile.

Paradójicamente, al mismo tiempo que muchos profesionales de la salud se encontraban con un exceso de trabajo en el cuidado de pacientes con COVID-19, otros se encontraban en una posición de insuficiente trabajo dado el aplazamiento generalizado de la consulta ambulatoria y procedimientos electivos. $\mathrm{Si}$ bien el objetivo principal era liberar recursos y limitar la propagación de COVID-19, estas medidas impusieron una carga financiera considerable tanto al sistema de salud como a los profesionales que trabajan de forma independiente. En este estudio observamos que aproximadamente un $75 \%$ de los profesionales se desempeñaban parcial o exclusivamente en el sistema privado de salud, lo cual explica el gran impacto económico que causó la pandemia, con un $77 \%$ de los encuestados que trabajaban de forma exclusiva o parcial en el sistema privado reportando una disminución del 50\% o más de su ingreso mensual, asociado a que un $36,9 \%$ del total de nuestra muestra tuvo que recurrir a alguna ayuda financiera durante la pandemia. En EE. UU. los ingresos de los profesionales de la salud disminuyeron aproximadamente un 50\% en marzo y abril de 2020, en comparación con el mismo período de $2019^{35}$.

Una de las limitaciones inherentes a nuestro estudio es el método de recolección de datos. Se optó por esta metodología dado las dificultades propias de la pandemia, con las medidas de distanciamiento social presentes al momento de realizar la encuesta. Por otro lado, esta encuesta no incluyó a todos los otorrinolaringólogos de Chile, dado que fue difundida a los afiliados a la SOCHIORL que corresponden a aproximadamente el $80 \%$ de los otorrinolaringólogos inscritos en la superintendencia de salud. Por otra parte, la encuesta se realizó al inicio de la pandemia en un período acotado de tiempo, si bien no fue el período de más contagio en Chile, correspondió al período de mayor incertidumbre y restricciones.

\section{Conclusión}

La pandemia por COVID-19 ha tenido un gran impacto en la práctica otorrinolaringológica en Chile, con una reducción drástica de la consulta ambulatoria y la actividad quirúrgica e importantes consecuencias económicas. Llama la atención el desarrollo de la telemedicina como consecuencia positiva, especialmente útil para lugares aislados del país. Aún seguimos luchando contra esta pandemia, por lo que el verdadero impacto sólo se podrá esclarecer con el tiempo, sin embargo, el conocer estos resultados significa un avance, ya que nos permitirá buscar medidas para mitigar este impacto negativo en el futuro.

\section{Agradecimientos}

A la SOCHIORL por colaborar con la difusión de la encuesta. 


\section{Bibliografía}

1. Zhou P, Yang XL, Wang XG, et al. A pneumonia outbreak associated with a new coronavirus of probable bat origin. Nature. 2020;579:270-273. doi: 10.1038/s41586-020-2012-7.

2. Organización Mundial de la Salud. COVID-19: cronología de la actuación de la OMS. Disponible en: https://www.who.int/es/news-room/detail/27-042020-who-timeline---covid-19. Consultado el: 30 de mayo de 2020.

3. World Health Organization. COVID-19 Weekly Epidemiological Update - 5 January 2021. Disponible en: https://www.who.int/publications/m/item/ weekly-epidemiological-update---5-january-2021. Consultado el: 20 de junio de 2021.

4. Goyal P, Choi JJ, Pinheiro LC, et al. Clinical Characteristics of Covid-19 in New York City. N Engl J Med. 2020;382:2372-2374. doi: 10.1056/ NEJMc2010419.

5. Guan W, Ni Z, Hu Y, et al. Clinical characteristics of coronavirus disease 2019 in China. N Engl J Med. 2020;382:1708-1720. doi: 10.1056/NEJMoa2002032.

6. Lechien JR, Chiesa-Estomba CM, De Siati DR, et al. Olfactory and gustatory dysfunctions as a clinical presentation of mild-to-moderate forms of the coronavirus disease (COVID-19): a multicenter European study. Eur Arch Otorhinolaryngol. 2020;277:2251-2261. doi: 10.1007/s00405-020-059651.

7. Stawicki SP, Jeanmonod R, Miller AC, et al. The 2019-2020 novel coronavirus (severe acute respiratory syndrome coronavirus 2) pandemic: A joint american college of academic international medicine-world academic council of emergency medicine multidisciplinary COVID-19 working group consensus paper. J Glob Infect Dis. 2020;12:4793. doi: 10.4103/jgid.jgid_86_20.

8. van Doremalen N, Bushmaker T, Morris DH, et al. Aerosol and Surface Stability of SARS-CoV-2 as Compared with SARS-CoV-1. N Engl J Med. 2020;382:1564-1567. doi: 10.1056/NEJMc2004973.

9. Wang J, Zhou M, Liu F. Reasons for healthcare workers becoming infected with novel coronavirus disease 2019 (COVID-19) in China. J Hosp Infect. 2020;105:100-101. doi: 10.1016/j.jhin.2020.03.002.

10. Wu Z, McGoogan JM. Characteristics of and Important Lessons from the Coronavirus Disease 2019 (COVID-19) Outbreak in China: Summary of a Report of 72314 Cases from the Chinese Center for Disease Control and Prevention. JAMA. 2020;323:1239-1242. doi: 10.1001/jama.2020.2648.

11. Centers for Disease Control and Prevention. Cases \& Deaths among Healthcare Personnel. Disponible en: https://covid.cdc.gov/covid-data-tracker/\#healthcare-personnel. Consultado el: 25 de julio de 2021.

12. GIMBE. Pandemia Coronavirus e campagna vaccinale. Disponible en: https://coronavirus.gimbe. org/. Consultado el: 31 de mayo de 2020.

13. Red Nacional de Vigilancia Epidemiológica. Análisis de Los Casos de COVID-19 En Personal Sanitario Notificados a La RENAVE Hasta el 10 de mayo en España. 2020. Disponible en: https://www.isciii.es/QueHacemos/ Servicios/VigilanciaSaludPublicaRENAVE/ EnfermedadesTransmisibles/Documents/ INFORMES/Informes\%20COVID-19/ COVID-19\%20en\%20personal\%20sanitario $\% 20$ 29\%20de\%20mayo\%20de\%202020.pdf

14. Zou L, Ruan F, Huang M, et al. SARS-CoV-2 viral load in upper respiratory specimens of infected patients. N Engl J Med. 2020;382:1177-1179. doi: 10.1056/NEJMc2001737.

15. Mick P, Murphy R. Aerosol-generating otolaryngology procedures and the need for enhanced PPE during the COVID-19 pandemic: A literature review. J Otolaryngol Head Neck Surg. 2020;49:29. doi: 10.1186/s40463-020-00424-7.

16. ENTUK. Guidelines for changes in ENT during COVID-19 Pandemic. Disponible en: https://www. entuk.org/entuk-guidelines-changes-ent-duringcovid-19-pandemic Consultado el: 31 de mayo de 2020.

17. Lu D, Wang H, Yu R, Yang H, Zhao Y. Integrated infection control strategy to minimize nosocomial infection of coronavirus disease 2019 among ENT healthcare workers. J Hosp Infect. 2020;104:454-455. doi: 10.1016/j.jhin.2020.02.018.

18. Alobid I, Cabrera P, Díaz de Cerio P, López F, Parente P. Recomendaciones de la SEORL-CCC para la práctica de la especialidad durante la pandemia de COVID-19. SEORL-CC. Disponible en: https://seorl. net/wp-content/uploads/2020/03/Recomendacionesde-la-SEORL-CCC-22-de-marzo-de-2020.pdf.

19. Sociedad Chilena de Otorrinolaringología Medicina y Cirugía de Cabeza y Cuello. Recomendaciones de la Sociedad Chilena de Otorrinolaringología y Cirugía de Cabeza y Cuello para el ejercicio de la especialidad durante la pandemia COVID-19 (SARS-CoV-2). Disponible en: https://sochiorl.cl/web/imgnuevas/ videos_covid/RECO_MEDICOS.pdf.

20. Lescanne E, van der Mee-Marquet N, Juvanon JM, et al. Best practice recommendations: ENT consultations during the COVID-19 pandemic. Eur Ann Otorhinolaryngol Head Neck Dis. 2020;137:303308. doi: 10.1016/j.anorl.2020.05.007.

21. Bann DV, Patel VA, Saadi R, et al. Impact of coronavirus (COVID-19) on otolaryngologic surgery: Brief commentary. Head Neck. 2020;42:1227-1234. doi: 10.1002/hed.26162.

22. Vukkadala N, Qian ZJ, Holsinger FC, Patel ZM, Rosenthal E. COVID-19 and the Otolaryngologist: Preliminary Evidence-Based Review. Laryngoscope. 2020;130:2537-2543. doi: 10.1002/lary.28672. 
23. Kowalski LP, Sanabria A, Ridge JA, et al. COVID-19 pandemic: Effects and evidence-based recommendations for otolaryngology and head and neck surgery practice. Head Neck 2020;42:1259-1267. doi: 10.1002/hed.26164.

24. Givi B, Schiff BA, Chinn SB, et al. Safety Recommendations for Evaluation and Surgery of the Head and Neck during the COVID-19 Pandemic. JAMA Otolaryngol Head Neck Surg. 2020;146:579-584. doi: 10.1001/jamaoto.2020.0780.

25. Ministerio de Salud de Chile. Resolución exenta No 156. Diario Oficial de la República Chile 2020 abril $1 ; \mathrm{I}: 1-5$.

26. Subsecretaría de Redes Asistenciales Gobierno de Chile. Ordinario C27 No808 2020 marzo 27.

27. Hussaini AS, Clark CM, Patel AA, et al. Management of Adult Inpatient Otolaryngologic Consultations During the COVID-19 Pandemic: A Proposed TierBased Triage System. Otolaryngol Head Neck Surg. 2020;163:330-334. doi: 10.1177/0194599820931011.

28. Sociedad Española de Otorrinolaringología y Cirugía de Cabeza y Cuello. Resumen de resultados de la encuesta actividad ORL durante la crisis por COVID-19. Disponible en: https://seorl.net/ wp-content/uploads/2020/04/RESULTADOSENCUESTA-ORL-CRISIS-COVID-19.pdf.

29. Kasle DA, Torabi SJ, Savoca EL, Judson BL, Manes
RP. Outpatient Otolaryngology in the Era of COVID-19: A Data-Driven Analysis of Practice Patterns. Otolaryngol Head Neck Surg. 2020;163:138144. doi: 10.1177/0194599820928987.

30. Mannelli G, Ralli M, Bonali M, et al. Impact of COVID-19 pandemic on Italian otolaryngology units: A nationwide study. Acta Otorhinolaryngol Ital. 2020;40:325-331. doi: 10.14639/0392-100X-N0832.

31. Ministerio de Salud de Chile. Resolución Exenta No 203. Diario Oficial de la República de Chile 2020 marzo 25;I:1-6.

32. Coca-Pelaz A, Takes RP, Hutcheson K, et al. Head and Neck Cancer: A Review of the Impact of Treatment Delay on Outcome. Adv Ther. 2018;35:153-160. doi: 10.1007/s12325-018-0663-7.

33. Ning AY, Cabrera CI, D'Anza B. Telemedicine in Otolaryngology: A Systematic Review of Image Quality, Diagnostic Concordance, and Patient and Provider Satisfaction. Ann Otol Rhinol Laryngol. 2020;130:195-204. doi: 10.1177/0003489420939590.

34. Paderno A, Fior M, Berretti G, et al. SARSCoV-2 Infection in Health Care Workers: Crosssectional Analysis of an Otolaryngology Unit. Otolaryngol Head Neck Surg. 2020;163:671-672. doi: 10.1177/0194599820932162.

35. FAIR Health. Healthcare Professionals and the A Comparative Study of Revenue and Utilization; 2020. 